\title{
La Quarantaine à Malte aux XVIIe et XVIIIe siècles dans les mémoires, journaux et récits de quelques voyageurs
}

\section{Carmen Depasquale}

\begin{abstract}
French Travel Literature, Memoirs and Diaries on Quarantine in Malta, Seventeenth to early Nineteenth centuries

Malta, a tiny island in the middle of the Mediterranean Sea, dependent on sea commerce and therefore particularly vulnerable to contagious diseases, had every reason to ensure that strict health regulations concerning the entry of persons and vessels were drawn up and respected. The rigorousness of this regime is perhaps not surprising when one considers that from 1530 to 1798 the rulers of the Maltese archipelago were the Hospitaller Knights of St. John. Travellers spent a varying period of time, depending on their place of departure, in quarantine on Manoel Island. This article undertakes to study the reaction of travellers to these precautions, analysing their impressions and descriptions of how these days in quarantine were spent, limiting itself to authors who chose the French language as the medium of their travelogues, memoirs or diaries.
\end{abstract}

\section{Introduction}

\section{L'Ordre de Saint Jean de Jérusalem de Rhodes et de Malte}

Il est difficile d'imaginer quel aurait été le destin d'une île dont la superficie ne dépasse pas 314 kilomètres carrés, "terre $[\ldots]$ ingrate $[\ldots]$ aride $[\ldots]$ malheureuse ${ }^{1}$ » pour ne citer qu'un voyageur, si elle n'était pas située au milieu de la Méditerranée. Grâce à sa position géographique, l'archipel maltais ${ }^{2}$ est depuis la nuit des temps au carrefour de l'activité maritime du mare nostrum et au croisement des civilisations. L'île subit la domination de toutes les puissances européennes, et d'une domination arabe de plus de deux cents ans allant de 870 à 1090, jusqu'en 1964 où elle obtint son indépendance de la Grande Bretagne. En 1565, elle fut assiégée pendant quatre mois par Soliman le Magnifique mais elle n'eut pas le même destin que Rhodes. En effet, c'est après avoir perdu Rhodes en 1522 que l'Ordre de Saint Jean de Jérusalem et de Rhodes

1 Dominique Vivant Denon, Voyage en Sicile (Paris, 1993), 137.

2 L'archipel maltais comprend deux îles habitées, Malte et Gozo, une troisième île, Comino, située entre les deux et deux autres rochers inhabités, Cominetto et Filfla.

The Northern Mariner/le marin du nord, XIX No. 2, (April 2009), 149-170 


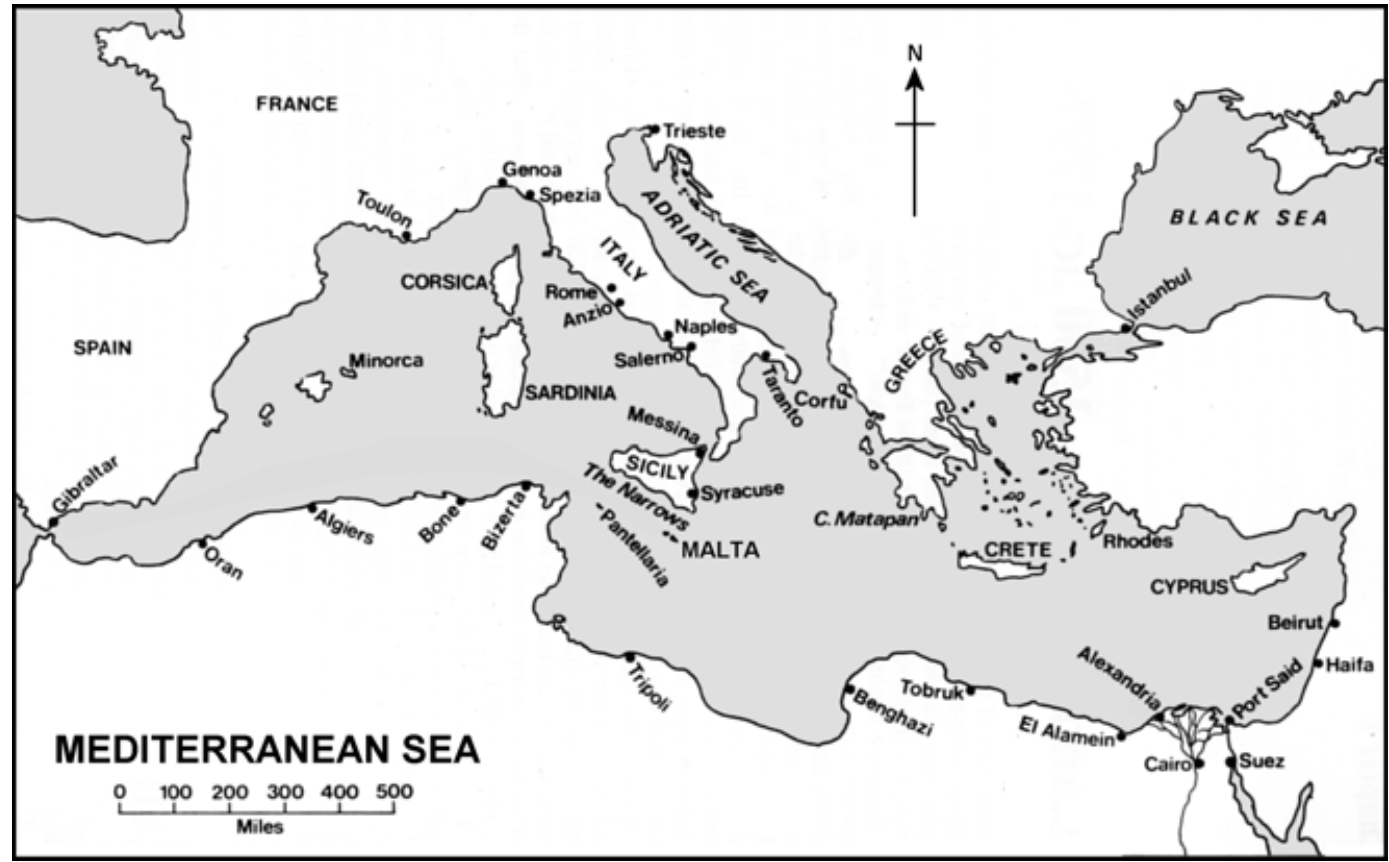

Illustration 1: The Mediterranean Sea. Archives and Collections Society.

accepta, huit ans après, cette île stérile que lui offrait Charles Quint. Malte devint alors le siège de l'Ordre de Saint Jean de Jérusalem, de Rhodes et de Malte, désignée, après 1530, par l'appellation l'Ordre de Malte, et y resta 268 ans, de 1530 à 1798.

Cet Ordre, composé de la fine fleur de la noblesse européenne, était divisé en huit Langues dont trois françaises: Provence, Auvergne et France. Les autres Langues étaient : Italie, Allemagne, Aragon, Castille et Angleterre. Cette dernière ne donnant plus de chevaliers après la Réforme - car il fallait être catholique pour y être admis - fut remplacée en 1782 par la langue anglo-bavaroise. Malte était donc pendant cette longue période une sorte d'union européenne, comme le constate en 1770 le voyageur britannique Patrick Brydone :

Comme Malte est un abrégé de l'Europe et un assemblage des cadets de famille des meilleures maisons, c'est sûrement une excellente académie de politesse [...]. La communication et la familiarité adoucissent et dissipent peu à peu les ridicules et les préjugés de chaque nation. Il est curieux de remarquer l'effet que produit ce mélange sur des hommes de différentes contrées ${ }^{3}$.

L'Ordre de Malte doit ses origines à des marchands d'Amalfi qui, en 1048, firent construire à Jérusalem d'abord un monastère et une auberge, ensuite un hôpital, dans le but de se charger des pèlerins chrétiens. En 1113, l'Ordre fut reconnu par le pape Pascal

3 Patrick Brydone, Voyage en Sicile et à Malte, traduit de l'anglais par M. Demeunier (2 édition, Amsterdam, Paris, 1776), 369-370. 
II. Son chef était Gérard de Martiques, dit Gérard Tenque. Ses nouveaux statuts, confirmés par le pape Calixte II, en firent un ordre chevaleresque, monastique, hospitalier et militaire. En 1128, les membres de cet Ordre prirent le nom de chevaliers de Saint Jean de Jérusalem. Le titre de Grand Maître fut conféré par le pape Clément IV en 1276 à leur chef, Hugues de Revel. Avec la défaite des chrétiens à Acre en 1291, l'Ordre fut obligé de quitter Jérusalem. Il se retira d'abord à Chypre, puis quinze ans après, il s'installa à Rhodes. L'Ordre administra à Rhodes un Etat souverain très bien organisé jusqu'en 1522.

Lorsque l'Ordre s'installe à Malte en 1530, son premier souci est de fortifier l'île. Le Grand Maître, Jean de La Valette commence, en 1566, la construction d'une ville fortifiée, La Valette, qui porte toujours son nom. Les Grands Maîtres successifs continuent à fortifier l'île le long de la côte. Malte devient un état souverain et l'Ordre a des ambassadeurs dans les villes principales d'Europe. La cour du Grand Maître devient celle d'un Prince symbolisée par la couronne fermée qu'introduit au XVIII ${ }^{e}$ siècle le Grand Maître Emmanuel Pinto de Fonseca (1741-1773).

Pour être reçu chevalier, des commissaires sont nommés pour l'examen des preuves de noblesse. Reçu dans l'une des Langues, le chevalier vient faire deux ans de noviciat à Malte, généralement à l'âge de seize ans. Il s'agit de la préparation religieuse, hospitalière et militaire du jeune homme. Celle-ci comprend quatre courses en mer. Le noviciat accompli, le jeune chevalier prononce ses vœux lors d'une cérémonie qui se tient dans l'église conventuelle dédiée au patron de l'Ordre, Saint Jean Baptiste. Le chevalier profès peut choisir de faire carrière dans l'Ordre en occupant des charges importantes. L'Ordre possède des commanderies et des biens-fonds dans tous les royaumes catholiques: ils constituent la source principale de ses revenus. En obtenant une commanderie, le chevalier prend le titre de commandeur. Quelques commandeurs avancent encore et sont nommés baillis. C'est parmi ces derniers qu'est élu le Grand Maître à la mort du précédent. Le Grand Maître gouverne avec son Conseil. Sont aussi membres de l'Ordre les chapelains conventuels qui sont chargés de desservir l'église conventuelle et les autres églises de l'Ordre, ils sont aussi aumôniers des hôpitaux, des vaisseaux et des galères; et les frères servants d'armes qui servent sous les ordres des chevaliers ${ }^{4}$. Le Grand Maître avait aussi l'autorité de nommer chevalier de grâce n'importe qui aurait été utile à l'Ordre, comme les peintres Michelangelo Merisi, dit le Caravage, pour ses tableaux destinés à l'église conventuelle ${ }^{5}$, et Antoine de Favray qui, de 1744 jusqu'à sa mort en 1798, était le peintre officiel de la cour magistrale.

La marine de l'Ordre était renommée en Europe : c'était là que se formèrent plusieurs marins, français et italiens surtout, qui passaient ensuite au service de leur pays $^{6}$. Les galères maltaises faisaient continuellement la police de la Méditerranée pour

4 Les chapelains conventuels et les servants d'armes ne devaient pas fournir de preuves de noblesse.

5 Le Caravage (1569-1609) fut fait chevalier de grâce le 14 juillet 1608 , mais le $1^{\text {er }}$ décembre de la même année cet honneur lui fut enlevé à cause de ses mœurs.

6 Parmi les Français, il serait à propos de signaler le bailli Pierre-André de Suffren (17291788) qui remporta une série de victoires au large des Indes (1782-1783) jusqu'à la signature 


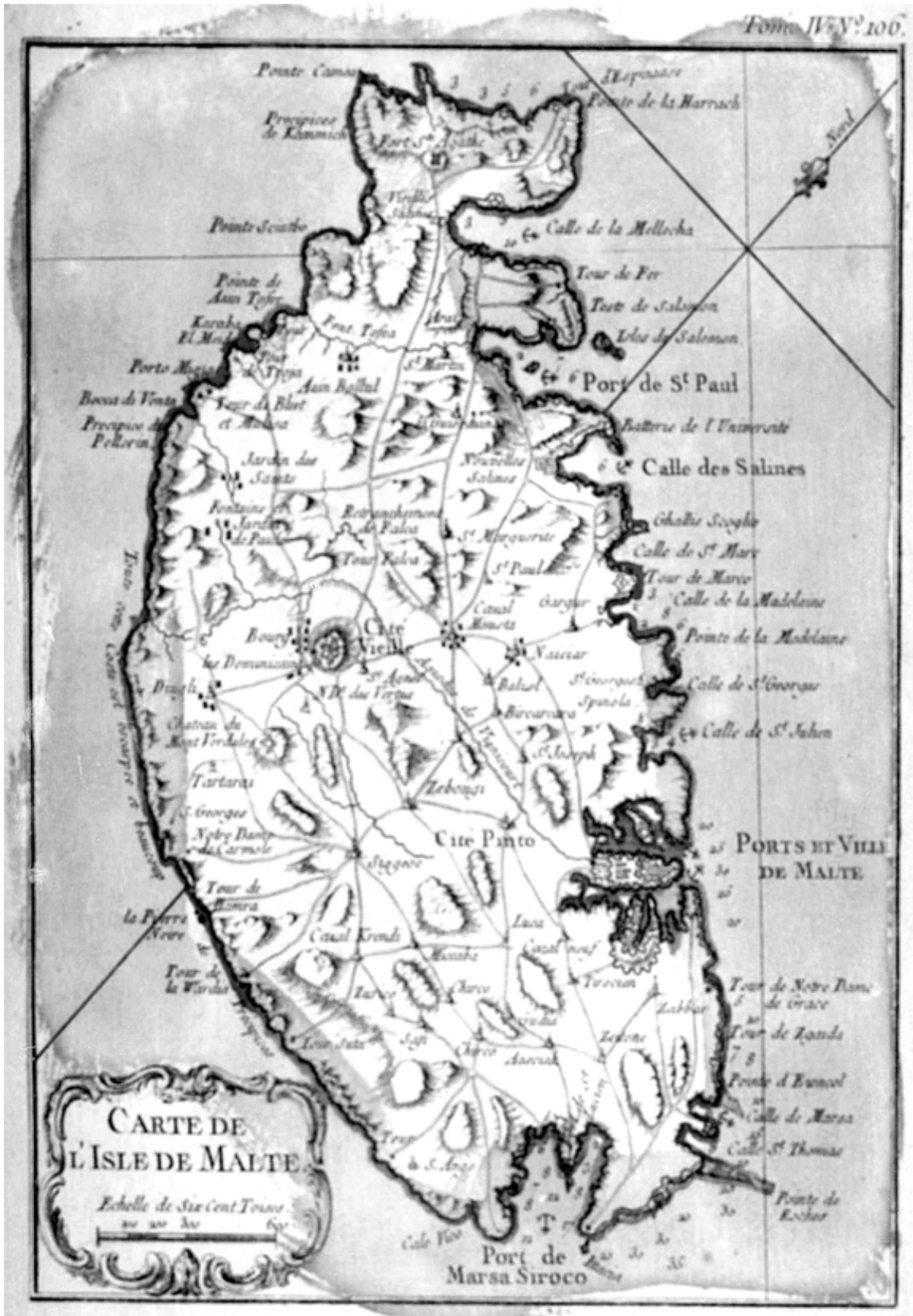

Illustration 2: Carte de l'Isle de Malte. Anon. France mid-18th century, from a multi-volume atlas and identified as Vol. IV, No.106. South West at top. Courtesy University of Malta Library.

de la paix de Versailles en 1783. 
sauvegarder le commerce des nations chrétiennes contre les pirates barbaresques. Par conséquent, les voyageurs qui découvraient l'Italie, la Sicile, la Grèce et le Levant trouvaient facilement le moyen de faire étape à Malte. D'autres qui ne voyageaient pas pour le plaisir, comme l'ambassadeur de France à Constantinople, s'arrêtaient souvent à Malte lorsqu'ils prenaient la voie de la mer. Enfin, une autre catégorie de voyageurs - les corsaires berbères dont la Méditerranée était infestée - eux aussi arrivaient à Malte malgré eux lorsque, au cours de la guerre de course, ils rencontraient les vaisseaux de l'Ordre qui, selon les règles, étaient « employés perpétuellement à purger la Méditerranée des corsaires dont elle [était] infectée ${ }^{7} »$. D'ailleurs, les instructions du Grand Maître aux capitaines des galères étaient claires : il fallait corseggiare [...] per fare qualche buon buttino e ricca presa ${ }^{8} »$. Le butin comprenait objets et hommes : ceux-ci devenaient esclaves : les plus robustes ramaient dans les galères, les autres étaient vendus au marché public aux particuliers. La vocation militaire de l'Ordre, celle de « protéger le commerce de la Méditerranée contre les rapines des Barbaresques ${ }^{9} »$ n'était donc pas privée de considérations économiques ${ }^{10}$.

\section{Commerce et contagion}

Si la mer était source de richesse, qu'elle liait Malte au continent, porteuse de lettres et de nouvelles, "Malte étant le rendez-vous général de tous les vaisseaux du Ponant et du Levant» selon un voyageur anonyme du XVII ${ }^{\mathrm{e}}$ siècle ${ }^{11}$, elle était aussi porteuse de contagion, au moins pouvait-elle l'être. Malte servait, au XVIII ${ }^{\mathrm{e}}$ siècle, " de dépôt de marchandises entre l'Orient et l'Occident ${ }^{12}{ }^{\prime}$ pour toute la Méditerranée, et ce, sans compter ses propres importations qui étaient nombreuses et ses quelques exportations. C'est précisément dans le but de faire de Malte un entrepôt qu'en 1723 fut promulgué la pragmatique du Grand Maître Antoine Manoel de Vilhena. Alain Blondy affirme : «Du Levant arrivèrent les grains, les dattes, les figues, les raisins secs, l'huile, le coton, la soie, le lin, le cordage, le cuir (brut ou travaillé), le bois de buis ; d'Occident vinrent les grains, le bois à brûler, les draps, la laine, les produits manufacturés. Malte devint un grand centre d'échanges, au point que les magasins et

$7 \quad$ Luc de Boyer d'Argens, Réflexions politiques sur les devoirs des chevaliers de Malte (La Haye, 1739), 108.

8 C'était l'expression utilisée dans les instructions que donnait le Grand Maître chaque fois que l'escadre de l'Ordre sortait en croisière.

9 Ce mot est du chevalier Etienne-François Turgot (1721-1798) auteur du manuscrit, National Library of Malta [NLM], Manuscripts [Libr] 262, Projet du chevalier de Turgot pour engager les puissances en guerre avec les Barbaresques à donner des subsides à la Religion [autre appellation pour l'Ordre de Malte].

10 Pour des renseignements plus détaillés sur l'Ordre de Malte et ses activités maritimes, voir Carmen Depasquale, « Voyageurs, corsaires, caravanistes : aventures en mer, spectacle dans les ports », The Northern Mariner/Le Marin du Nord XV No. 2 (April 2005), 19-41.

11 Anonyme, Nouvelle relation du voyage et description exacte de l'isle de Malthe (Paris, 1679), 159.

12 Jacques Godechot, «La France et Malte aux XVIII ${ }^{\mathrm{e}}$ siècle », Revue historique ( juilletseptembre, 1951), 72. 


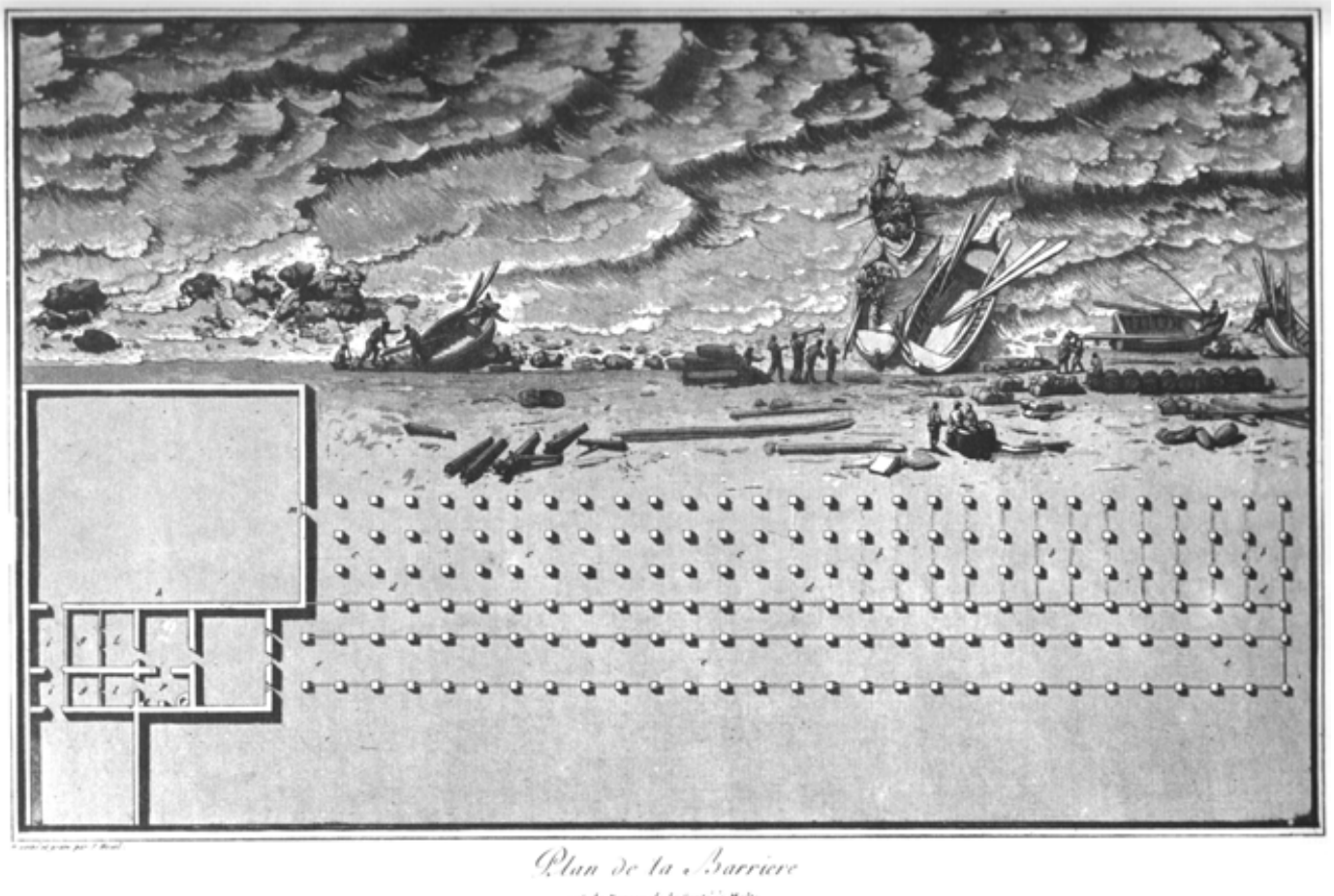

Illustration 3: Plan de la Barrière, planche CCLIV du Vol IV de Jean Houel, Voyage pittoresque des îles de Sicile, de Malte et de Lipari, (Paris, 1782). Avec l'aimable autorisation du Director, University of Malta Library, Msida, Malta

entrepôts du quai de la Barrière ${ }^{13}$ s'avérèrent insuffisants. En 1752, Pinto décida de faire construire [...] dix-neuf magasins sur le quai de la Grand Marse ${ }^{14}$. $\gg$ Dans son excellent Corsairing to Commerce, Carmel Vassallo trace l'évolution du commerce du coton entre Malte et la péninsule ibérienne. Parmi les statistiques citées par Vassallo se trouvent celles qui donnent le nombre de Maltais commerçant dans la péninsule ibérienne de 1699 à 1761 : 184 en 1699, 236 en 1745/46, 374 en 1756, 528 en 1759, 484 en 1761, et en Espagne : 882 en 1772 et 1229 en $1791^{15}$. Le coton maltais était aussi exporté à Marseille. D'autre part, l'historien Victor Mallia-Milanes étudie le commerce entre Venise et Malte: «Large assortments of commodities - from timber, wax, and ironmongery to paper, copper, mirrors and other glassware - were shipped from the

13 Voir la note 23.

14 Alain Blondy, L'Ordre de Malte au XVIII' siècle (Paris, 2002), 46-47.

15 Carmel Vassallo, Corsairing to Commerce (Malta, Malta University Publishers, 1997). Dans sa Préface, xiv, Vassallo constate: "Consisting initially of the organisation of brigantine expeditions which apparently lasted up to the mid 1760s, it [Spanish trade] subsequently evolved into two separate (albeit not mutually exclusive) branches, which lasted into the early years of the nineteenth century. One was the practical monopoly of the supply of cotton yarn to Catalonia's cotton industry and the other was an amazing retail network which covered large areas of eighteenth century Spain. » 
Adriatic port [Venice] to Malta, very often in exchange for ashes of Kalimagnum. This commercial service was long standing. Vast shipments of foodstuffs and other products were purchased from Levantine markets, whereas it was only recently that the Maltese merchant had been venturing on Dalmation markets, particularly for wine. " Le même auteur constate l'établissement d'un consulat maltais à Venise dans les années 1750 et fait référence au "lucrative bilateral commercial agreement concluded between the two countries in the $1760 s^{16}$.» Alain Blondy signale le commerce du vin: «En 1723, il y avait 28 négociants importateurs et 5 patrons de bateaux spécialisés dans le commerce du $\operatorname{vin}^{17}$.» Autre source de témoignage de cette activité portuaire nous est donnée par les récits des voyageurs qui ne manquent pas d'en d'être impressionnés et par maints peintres qui en font le sujet de leurs tableaux. Deux auteurs qui publient leurs récits anonymement donnent en grand détail une liste d'objets exportés et importés, soit pour la consommation locale, soit pour la réexportation, comme dans le cas des « rubis de Messine [qui] sont un commerce que les Grecs font par les Maltais dans le Levant ${ }^{18}$.» Cet auteur conclut : «Le débit considérable de provisions de [toute] espèce qu'entraine le grand nombre de navires qui fréquentent le port de Malte, donne un bénéfice aux marchands dont l'active intelligence met à profit le plus léger besoin des étrangers. On trouve à Malte des compagnies de spéculateurs qui assurent à un taux raisonnable les biens, la vie, la liberté. »Plus loin, il affirme : «L'île ne fournit pas à la nourriture des habitants qui tirent leurs grains, vins, bois, bétail de Sicile et de la Morée. L'Ordre a pour cet effet des magasins immenses tant à Malte qu'à Augusta, Palerme, Girgenti, Marseille, Venise, et pour la glace qui est de la première nécessité, le dépôt est à Riposto en Sicile $^{19}$.» Dans Malte par un voyageur français, l'auteur anonyme rappelle l'institution du Consolato del Mare: «Les causes relatives au commerce sont portées à un tribunal consulaire qu'institua en 1697, à la Cité Vallette, le Grand Maître Perellos [1697-1720] après avoir fait d'exactes recherches sur les usages des plus célèbres places de commerce $^{20}$.»

On ne s'étonne donc pas qu'à Malte circulaient pistoles d'Espagne, ducats de Venise, tarins siciliens, livres et louis français - toute cette monnaie était acceptée à Malte, même si l'Ordre frappait sa propre monnaie. Ce fait se vérifie non seulement dans les récits des voyageurs, tel celui de Roland de La Platière qui, après avoir décrit la monnaie en argent, en or et en cuivre frappée à Malte, constate qu' « en ce moment, les louis de France sont très abondants ${ }^{21} »$; ou celui du comte de Borch qui signale le commerce lucratif du «change des monnoyes étrangères que l'affluence de toutes les

16 Victor Mallia Milanes, Venice and Hospitaller Malta 1530-1798 (Malte, 1992), 225.

17 Blondy, L'ordre de Malte, 102.

18 Anonyme, Malthe, Corse, Minorque et Gibraltar, s.1., 1797.

19 Ibid., 72.

20 Anonyme [François-Emmanuel Guignard de Saint-Priest], Malte par un voyageur français (Malte, 1791), 46.

21 Jean-Marie Roland de la Platière, Lettres écrites de Suisse, d'Italie, de Sicile et de Malte (1776-1778) (Amsterdam, 1780), vol. III, 88-89. 
nations y [à Malte] apporte en abondance ${ }^{22} »$, mais aussi dans la lecture d'une comédie manuscrite du chevalier Joseph de Fassion de Sainte-Jay où le commandeur Cliton, parlant de l'argent qu'il doit à ses créanciers, cite écus, séquins et pistoles dans la même scène ${ }^{23}$.

Dès son arrivée à Malte, l'Ordre de Saint Jean, fidèle à sa vocation hospitalière, établit l'Infirmerie. Avec la construction de la nouvelle ville, la Sacrée Infirmerie qui existe toujours fut l'un des premiers bâtiments. Elle accueillait tous les malades sans distinction de classe ni de religion. Les lits étaient individuels et les salles étaient divisées selon les maladies: maladies fébriles, blessures, interventions chirurgicales, dysenterie, maladies mentales, maladies contagieuses (tuberculose) ou vénériennes. Il y avait un autre hôpital pour les femmes, un autre pour les enfants trouvés et le Lazaret. Le service était effectué par les chevaliers et le Grand Maître lui-même rendait visite aux malades tous les vendredis. Médecins, chirurgiens barbiers et apothicaires, nommés et salariés par l'Ordre, devaient se conformer à des obligations précises. Ils faisaient leurs études à l'école de médecine à Malte et spécialisaient dans les domaines différents à Paris, à Montpellier et à Florence. L'expertise des chirurgiens maltais était recherchée à l'étranger, par le Bey de Benghazi et la république de Venise, par exemple ${ }^{24}$.

L'Ordre hospitalier de Saint Jean savait plus que n'importe qui combien il était facile d'importer les maladies contagieuses si on négligeait de prendre toutes les précautions. Furent rédigées à cette fin des lois sévères qui assuraient que seulement les personnes à qui on donnait libre pratique pouvaient parcourir l'île et se mêler aux habitants. Cet article traitera des mesures prises par l'Ordre pour s'assurer que la population maltaise n'en fut pas victime. L'historien de la médecine à Malte, Paul Cassar, constate que des règlements concernant la quarantaine, c'est-à-dire une période d'isolement pour hommes, vaisseaux et marchandises qui arrivaient à Malte de l'étranger, étaient déjà en place au Moyen Age. Le port de Marsamxett servait de port de quarantaine ${ }^{25}$. Cependant, deux ambassadeurs de Sa Majesté britannique à Constantinople qui s'étaient arrêtés à Malte en route pour la Grande Bretagne en avril 1669 et en 1686 passèrent leur quarantaine dans une villa dans le faubourg de la Valette, à Floriana ${ }^{26}$. Le lazaret trouva sa place permanente en $1643^{27}$ sur une petite île où le Grand

22 Michel Jean, comte de Borch, Lettres sur la Sicile et sur l'île de Malthe (Turin, 1782), 198.

23 Joseph de Fassion de Sainte-Jay, Le Commandeur dans sa famille, éd. Carmen Depasquale (Malte, 2005), 88-89.

24 Voir NLM, Archives of the Order of Malta [Arch] 1713, 6, Arch 1511, 140, Arch 1504, 124, Libr 1146, vol II, 72.

25 Le lazaret était d'abord situé de l'autre côté du port à Rinella, ensuite dans la partie de la Valette appelée encore aujourd'hui «la Barrière » à cause de la barrière qui séparait les voyageurs en quarantaine des habitants de l'île. Voir aussi la note 39.

26 Voir Paul Cassar, Medical History of Malta (London, 1964), 299.

27 NLM, Arch 114; NLM, Libr 1146, vol. 1; Bartolomeo Dal Pozzo, Historia della Sacra Religione Militare di S. Giovanni (Venezia, 1715), 76. Selon Cassar, Medical History, 299300, le lazaret à La Valette ne cessa pas de fonctionner: It seems that the Lazaretto at Valletta was kept in use for quite a long time after the erection of the new building in Manoel 
Maître Manoel de Vilhena fit construire un fort en 1726 ; depuis, l'île prit le nom de ce Grand Maître, Manoel Island. La durée de la quarantaine variait selon le cas, allant de quelques jours jusqu'à l'imposition de la double quarantaine ${ }^{28}$.

Quand l'Ordre s'installa à Malte furent appliquées les lois et procédures qu'il avait pratiquées à Rhodes et qui étaient modifiées en conformité avec les progrès faits dans le domaine de la médecine. Pourtant, ces précautions se vérifièrent insuffisants au temps du Grand Maître cardinal, Hugues de Verdalle. La peste éclata à Malte en 1592 et de nouveau en 1593. Elle fut importée d'Alexandrie en mai 1592 par quatre galères du Grand Duc de Toscane qui entrèrent dans le port de Malte après avoir capturé

deux navires venant d'Alexandrie richement chargés d'une grande quantité de riz, d'épices et de lin et fait cent cinquante esclaves turcs. Or, la peste régnait dans ce port d'Egypte et les galères toscanes furent infectées. Entre le Cap St. André [Cap le plus oriental de Chypre] et Malte, vingt-deux personnes moururent [...]. Alors que ces décès auraient dû affoler les autorités portuaires, les règles de santé de cette époque n'étaient aucunement strictes et chacun put descendre à terre ${ }^{29}$. En effet, en raison des habitudes de cette époque, la pratique fut donnée au bâtiment après deux jours seulement de station dans le port. Pire, les gens malades furent admis à la Sacrée Infirmerie. Là [...] les médecins $[\ldots]$ donnèrent l'alarme et déclarèrent le danger de peste, mais c'était trop tard [...]. La contagion avait été surtout occasionnée par l'arrivée des malades des galères à l'Infirmerie et par la vente du lin infecté, acheté dans le pays et donné partout à filer aux paysannes ${ }^{30}$.

On crut la peste disparue en septembre de la même année, elle réapparut le 12 novembre pour - à ce qu'on croyait - disparaitre en fin janvier 1593 ; une fois on recommença à commercer librement, elle éclata de nouveau deux mois plus tard pour ne disparaître complètement qu'en fin décembre 1593. "La peste avait fait 3600 morts parmi une population diminuée par la peste, l'année précédente, de 3000 personnes.

Island. As late as 1779-88 allusion is made in the Treasury accounts of the Government to " the expense of the considerable repairs made at the quarantine at the Grand Port ». En effet, c'est le lazaret de La Valette, la Barrière, que décrit et dessine Jean Houel. A ce sujet, voir plus loin.

28 Il n'était pas rare qu'on imposait une double quarantaine qui allait jusqu'à 40 jours pour les passagers et 80 jours pour la cargaison. Voir NLM, Arch 6527, 64, 88, 190 ; Arch 6528, 479, 516, 596.

29 Voir Cassar, Medical History, 298. Un voyageur anglais George Sandys qui s'arrêta à Malte en rentrant en Angleterre d'un voyage au Levant, commencé en 1610, raconte dans son journal qu'on le conduisit en bateau au Lazaret où on l'informa qu'il dut passer 30 ou 40 jours avant d'obtenir l'autorisation d'entrer en ville. Un capitaine français s'approcha de lui en bateau et lui offrit de l'amener en ville. L'Anglais refusa cette offre car il savait qu'il serait condamné à une mort certaine s'il désobéissait aux règles de la quarantaine. Le lendemain, le capitaine lui obtint la pratique, ce qui lui permit de quitter le lazaret. Cassar assure ses lecteurs qu'il s'agissait d'une exception, car les menaces constantes de la peste étaient telles que l'isolement des voyageurs était strictement observé. 
Quarante chevaliers étaient décédés. La Religion ${ }^{31}$ avait dépensé 27000 écus pour les soins donnés aux malades ${ }^{32} »$.

Ce n'est pas un cas isolé : la peste éclata à Malte de nouveau en 1676; la population fut décimée. En 1701 furent promulguées les Leggi e costituzioni prammaticali, suivies de la Pragmatique sanction de 1732, de plusieurs décrets ou bandi, pour être enfin incorporées en 1784 dans le code, Del Diritto Municipale di Malta, populairement appelé le code De Rohan.

\section{Le lazaret}

Le document de la Bibliothèque nationale de Malte qui porte la cote Libr 820, intitulé Registro della Sanità, fut rédigé par ordre du chevalier de Margon, commissaire de la santé. Y sont inscrits les noms des vaisseaux et passagers qui firent quarantaine à Malte du 22 mai 1723 au 11 septembre 1739. Les huit premiers feuillets non paginés font allusion à deux règlements ; le premier, Regolamento per la buona custodia della sanità, daté du 23 décembre 1701, traite des «nouvelles lois pragmatiques » que le Grand Maître venait de promulguer ${ }^{33}$. Le deuxième est le Regolamento fatto alli 12 settembre 1720 dalla Veneranda Deputatione della Sanità, con la partecipazione di Sua Eminenza per preservare col divino aiuto questa isola d'ogni mal epidemico. Il était interdit à toute personne de débarquer ou même de se transférer dans un autre vaisseau avant d'obtenir pratique des commissaires de la santé. Pour la peine, la loi distinguait entre les vaisseaux qui venaient des pays soupçonnés de contagion et les vaisseaux corsaires, et les autres con patente netta qui venaient des pays où il n'y avait pas de risque de contagion. Si le vaisseau venait d'un pays infecté, les transgresseurs, ainsi que le capitaine qui aurait permis ou ordonné une telle transgression, étaient condamnés à mort ; et ceux qui, en étant au courant, n'auraient pas déclaré le fait dans les vingt-quatre heures étaient condamnés aux galères à perpétuité. Dans les cas de transgressions concernant les vaisseaux venant de pays où on ne soupçonnait pas de contagion, la peine était de trois ans de galère ou d'une amende de trente onces ${ }^{34}$ pour les transgresseurs et le capitaine qui aurait permis ou ordonné la transgression, et d'un an de galère ou d'une amende de dix onces pour ceux qui n'auraient pas révélé ce dont ils étaient au courant dans les vingtquatre heures. Il était également interdit à toute personne sur terre d'accoster les vaisseaux qui venaient des pays soupçonnés de contagion ou les vaisseaux corsaires, sous peine d'être pendue. La nécessité de ces lois dérivait du fait qu'il s'agissait d'una materia di tanta rilevanza dalla quale dipende la conservazione della salute, non solo in questo dominio, ma in tutta Italia e in diversi altri stati della cristianita ${ }^{35}$. De surcroît, si le transgresseur était membre de l'Ordre il était privé de l'habit pour être jugé par le

$31 \quad$ Autre appellation pour l'Ordre de Malte.

32 Blondy, Hugues de Loubens de Verdalle, 179.

33 Voir NLM, Arch 265.

34 Monnaie de Sicile.

35 Il s'agissait «d'une matière d'une telle importance que c'est d'elle que dépend la préservation de la santé, non seulement dans ce pays, mais dans toute l'Italie et dans divers autres États de la chrétienté. » 
tribunal séculier.

La correspondance échangée de 1720 à 1722 entre l'Ordre et les autorités sanitaires de Toulon et de Marseille où il y avait la peste témoigne des mesures préventives prises par l'Ordre pour en empêcher l'« importation » à Malte. Dans ces lettres l'Ordre demanda aux autorités sanitaires de Toulon et de Marseille de lui fournir des renseignements précis sur l'épidémie dans le but de prendre les mesures nécessaires de quarantaine à l'égard des passagers et des marchandises arrivant à Malte de ces deux ports $^{36}$.

Voyageurs, équipage, marchandise ne quittaient donc pas le vaisseau avant que les autorités sanitaires portuaires ne donnassent pratique. On permettait aux vaisseaux infectés à ancrer dans des endroits spécifiques du port avec l'interdiction d'approcher d'autres vaisseaux ou de communiquer avec eux. Un décret de 1765 interdit aux pêcheurs de pêcher dans «les eaux de quarantaine ${ }^{37}$ ». La punition pour les prostituées était le bannissement, alors que les femmes ordinaires qui s'approchaient de ces vaisseaux étaient bannies des villes portuaires de la Valette, Birgu et Senglea pour cinq ans $^{38}$. Les vaisseaux de l'Ordre qui revenaient de la guerre de course avec des prises hommes et marchandises - étaient, eux aussi, assujettis à la quarantaine. S'il y avait des blessés, un chirurgien était transféré au lazaret où il devait rester jusqu'à la fin de la période de la quarantaine. Toute marchandise était désinfectée au lazaret. Les passagers et l'équipage étaient soumis à une dépurification au début de la quarantaine et encore deux jours avant sa fin. Personne n'était exempt de la quarantaine : ambassadeurs, ministres plénipotentiaires, consuls, chevaliers, tous devaient s'y soumettre. La purification de la marchandise, y compris celle in transit, consistait dans le fait qu'on l'exposait en plein air pendant trente-cinq jours après l'avoir déballée. On appliquait le même procédé aux vêtements et aux bagages des passagers ${ }^{39}$. Il y avait aussi au lazaret des étables pour l'isolement du bétail. Les vaisseaux étaient désinfectés de cette manière : la cale fut remplie d'eau de mer, ensuite lavée de vinaigre, une fois sèche elle était parfumée. Si dans les vaisseaux il y avait des malades qui avaient la peste, les autorités sanitaires leur refusaient l'entrée dans les ports, tout en leur fournissant des provisions d'eau, de biscuits et de légumes, ainsi que du vinaigre et de la paille nécessaires pour la dépurification du vaisseau. Dans les cas jugés extrêmes, le vaisseau était brûlé ${ }^{40}$. Un système de lettres circulaires entre les ports de Marseille, Syracuse,

36 Paul Cassar, French Influence on Medical Developments in Malta (Malta, 1987), 15.

37 NLM, Libr 429, 87.

38 NLM, Libr 740, 173.

39 NLM, Libr 19, 122.

40 Voir NLM, Arch 6530-6532. Voir aussi Paul Cassar, « Malta's Role in Maritime Health under the auspices of the Order of St John in the 18th century ", in The Lombard Bank (Malta) Limited, Annual Report 1989 (Malta, 1989). Dans cet article, l'auteur signale les documents d'archives de la NLM portant la cote Arch 6527-6533 qui traitent des vaisseaux, équipages, voyageurs, marchandises et bétail qui entraient dans le port de Malte de 1739 à 1801. Il ajoute que chaque entrée enregistre les renseignements suivants : le type du vaisseau et son nom, le nom et lieu de résidence du capitaine, le nombre de personnes, équipage et passagers, les ports de départ et de destination, la durée du voyage, le type et quantité de 
Messine, Naples, Venise et Malte assuraient que les nouvelles concernant les maladies contagieuses sévissant à Constantinople, Smyrne, Jaffa, Jérusalem et Alexandrie arrivaient dans ces ports ${ }^{41}$.

Ces documents nous éclairent aussi sur la durée de la quarantaine. Les vaisseaux certifiés con patente di sanità limpia [limpida, propre] subissaient une quarantaine de vingt-six jours, comme c'était le cas d'une martingane française qui entra dans le Lazaret le 20 mai 1723 et à qui on donna pratique le 16 juin. Le vaisseau de guerre de l'Ordre le Saint-Vincent commandé par le chevalier Jacques de Chambray qui avait capturé le 13 mai 1723 la Padrona di Tripoli avec 266 Turcs, 3 renégats et 32 esclaves chrétiens à qui fut rendue la liberté entra dans le port le 16 mai et obtint la pratique douze jours après ; on ne donna pratique aux esclaves turcs que le 31 mai. La quarantaine était de courte durée parce que la Padrona di Tripoli naviguait en mer déjà depuis 37 jours avant qu'elle ne fût prise : il n'y avait donc aucun danger de contagion. Au contraire, un vaisseau vénitien à la patente brutta [laide], arrivé à Malte le 14 août 1793, n'eut pratique que le 24 septembre suivant - quarantaine entière, quarante jours. Il faut tenir compte, cependant, que dans le cas où les occupants du vaisseau avaient passé déjà quelques semaines en mer ou qu'ils avaient fait quarantaine dans un autre port méditerranéen chrétien, ils ne faisaient pas la quarantaine à Malte.

Paul Cassar note qu'il n'y avait pas de lazarets en Grande Bretagne et que les vaisseaux en route pour l'Angleterre des ports du Levant étaient obligés à faire quarantaine à Malte ou dans un autre port de la Méditerranée avant de débarquer leur marchandise en Grand Bretagne ou en Irlande ${ }^{42}$. Le même auteur rappelle que les mesures adoptées par le système de quarantaine à Malte suivaient les théories épidémiologiques et les pratiques de l'époque, basées sur la doctrine de la 'contagion', c'est-à-dire que les maladies contagieuses étaient transmises directement par le contact de corps à corps ou indirectement par contact avec des objets appartenant aux malades ${ }^{43}$. Il conclut par cette évaluation de l'effet de ces mesures d'isolation prises par les autorités maltaises : "Though [...] the theory of " contagion » was wrong [...], the isolation of persons and objects at risk required by quarantine measures was not, after all, irrational and illogical but justifiable and sound. In applying this wise principle, therefore, the Malta quarantine system played a constructive role in protecting the health not only of the residents of the Maltese Islands but also of European ports and of countries bordering the Mediterranean from maritime invasions by plague thanks to the checks it imposed upon ships, cargos, crews and travellers ${ }^{44}$. » On admettait aussi au Lazaret tous les malades qui se trouvaient dans les vaisseaux. Selon Paul Cassar «la fièvre était souvent due à la malaria ou au typhus. D'autres souffraient de la dysenterie, du scorbut,

cargaison, la date d'accès des passagers et de débarquement de la marchandise au Lazaret, le nom des gardes sanitaires qui s'en occupent et la date de la pratique (fin de quarantaine).

41 Cassar, « Malta's Role », 15.

42 Cassar, Medical History, 289. L'auteur cite à son tour John Howard, An account of the principal lazarettos in Europe (London, 1789), 26.

43 Cassar, « Malta's Role», 21.

44 Ibid., 23. 
des maladies vénériennes et des cancers, d'autres encore avaient des blessures causées par la guerre de course ${ }^{45}$.

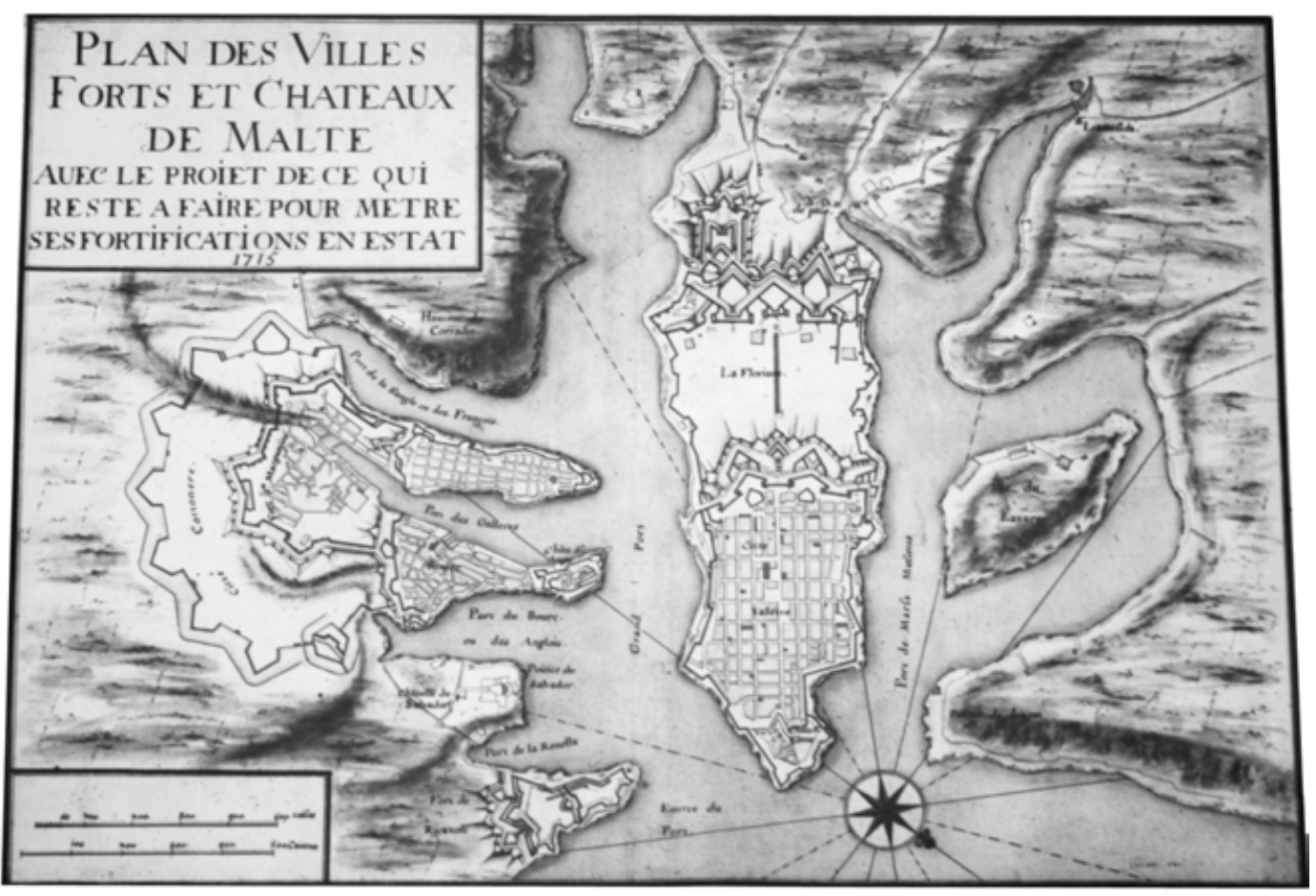

Illustration 4: map of the Grand Harbour showing Manoel Island, the Lazzaretto, on the right hand side. Courtesy of the Map Collection, National Library of Malta.

\section{Mémoires, journaux et récits de voyage}

Les documents d'archives ont servi aux historiens soit pour l'histoire de Malte, soit pour l'histoire de la médecine à Malte. Il en est de même pour les récits des voyageurs britanniques, écrits pour la plupart au $\mathrm{XIX}^{\mathrm{e}}$ siècle. Il existe une lacune pour ce qui est des récits de voyage écrits en français. Dans cette partie, on essaiera d'exposer comment la quarantaine était perçue ou vécue par ces derniers. Les ambassadeurs du roi de France à Constantinople s'arrêtaient à Malte lorsqu'ils prenaient la voie de la mer, mais souvent ils ne débarquaient pas à cause de certaines prétentions regardant le protocole ${ }^{46}$. C'était le cas du marquis de Ferriol (1698) et du marquis de Bonnac (1716), alors que le marquis de Castellane (1741) débarqua, mais garda l'incognito ${ }^{47}$. Par contre, le comte de Saint-Priest, en route pour Paris, débarqua à Malte le 6 octobre 1776, après

45 Ibid., 19.

46 Ces ambassadeurs avaient des instructions « de prendre la main sur le Grand Maître », alors que celui-ci voulait « faire cesser la prétention de la main sur le Grand Maître » (mémoire du Grand Maître daté du 13 juillet 1741). Voir Ministère des Affaires Etrangères [MAE], Mémoires et documents [MD] Malte 27, pièce no. 8, 96.

47 MAE, Correspondance Politique [CP] Malte 8, 339. 
un voyage de dix jours.

Il dit dans ses Mémoires qu'il passa « peu de jours » en quarantaine :

Je m'embarquai au mois d'octobre et me dirigeai sur Malte, où je voulais m'arrêter pour l'intérêt de mon jeune frère qui faisait alors ses caravanes. J'avais connu le Grand Maître de Rohan à Malte vingt ans auparavant. Nous étions tous deux alors simple chevaliers, mais l'année précédente, il avait été élu chef de son Ordre. Je subis dans l'île une quarantaine de peu de jours, elle n'eut rien de pénible. Mes anciens confrères eurent la bonté de venir me voir souvent et ma détention momentanée me parut moins longue par les soins qu'on voulut bien me rendre. Le Grand Maître résolut pourtant d'y mettre fin le jour qu'il devait prendre possession de la capitale de l'île, dite Cité Vieille [... $]^{48}$.

Ces «peu de jours » de quarantaine sont précisés dans une autre source, le journal de l'abbé Boyer qui donne la date de cette fête comme étant le 27 octobre et qui avait précisé la date de l'arrivée de l'ambassadeur, le 6 octobre :

M. de Saint-Priest, ambassadeur de France à Constantinople, et Madame son épouse sont arrivés en dix jours. Ils feront ici leur quarantaine et se rendront à Toulon sur la même frégate La Mignonne commandée par M. de Tramont. On dit que ce ministre a de grandes affaires à traiter à Versailles ${ }^{49}$.

Ces précisions nous permettent de constater que cette quarantaine dura vingt-six jours : seize à Malte, plus les dix jours en mer. Une autre précision révélée par le journal de Boyer est que la table de Madame de Saint-Priest était « servie par les officiers de bouche du palais » et que le temps qu'elle resta en quarantaine elle avait « travaillé un nœud d'épée » qu'elle présenta au Grand Maître lorsque celui-ci lui fit le compliment de lui rendre visite ${ }^{50}$.

En rappelant cette visite, le comte de Saint-Priest fait allusion au fait qu'il avait été chevalier de Malte, « reçu [dans la Langue de Provence] à l'âge de quatre ans ${ }^{51}$ ». En 1752, âgé de dix-sept ans, il quitta la France pour aller à Malte « faire ses caravanes [...], obligation indispensable $e^{52} »$. En effet, il avait été relevé de ses vœux ${ }^{53}$ pour pouvoir se marier, car son frère aîné était mort sans issue. Ce n'était donc pas son premier voyage à Malte, ni sa première expérience de quarantaine. Pourtant, combien le ton du récit de cette première expérience est différent! Cela va sans dire que la quarantaine subie la

48 François-Emmanuel Guignard comte de Saint-Priest, Mémoires, Règnes de Louis XV et de Louis XVI (Paris, 1921), 156. Le « jeune frère », Charles-Antoine, futur commandeur de Saint-Christol (1788), avait alors dix-huit ans, le frère aîné en avait quarante et un.

49 NLM, Libr 137, 194.

50 Ibid.

51 Saint-Priest, Mémoires, 3.

52 Ibid., 10. L'auteur explique que «les 'caravanes' étaient des croisières auxquelles tout chevalier de Malte était astreint de prendre part une fois dans sa vie. Elles avaient pour objet, le plus souvent, d'assurer la police des mers en faisant campagne contre les pirates barbaresques ». Avant sa profession, tout chevalier était censé faire quatre croisières.

53 Comme les membres de tout ordre monastique, les chevaliers prononçaient les vœux de chasteté, pauvreté et obéissance lors de la cérémonie de leur profession. 
première fois, qu'il définit par la phrase « quatorze jours de détention » était inattendue ! Si l'imposition de cette quarantaine provoqua l'incompréhension, voire la colère de la part du jeune homme impatient qui la subit, elle sert à montrer combien les autorités maltaises prenaient au sérieux le moindre risque de contagion.

Nous nous embarquâmes à Marseille [...]. Le huitième jour nous arrivâmes à Malte ; chacun s'attendait à entrer dans la ville, mais le capitaine du port nous signifia qu'on faisait faire quarantaine aux navires venant de Marseille, ce qui s'était établi sur un faux bruit de contagion. Il fallut subir quatorze jours de détention ; l'édifice destiné à cet objet était situé dans une petite rade vis-à-vis du grand port. La chaleur était suffocante ; mais comme il n'y avait que les quatre murailles, nous nous meublâmes des lits que nous avions sur notre vaisseau. Au reste les visites ne nous manquaient pas, à la distance respectueuse d'une vingtaine de pieds de roi [...]. Après notre quarantaine, nous nous établîmes dans la ville. ${ }^{54}$

Le voyage de Dominique Vivant Denon entre la Sicile et Malte s'avéra difficile à cause du mauvais temps. C'était la nuit du 5 au 6 septembre 1778. Il raconte un fait qui montre combien les autorités sanitaires prenaient au sérieux la possibilité d'une épidémie de peste. "L'impossibilité d'entrer à Malte la nuit, et mon impatience de mettre pied à terre », raconte-t-il, fit qu'il débarqua sur Comino,

petite île qui n'est qu'un rocher aride entre Goze et Malte [et qui ...] tient son nom du cumin qu'elle produit. [Après] à peine deux heures [...] je me sentis réveillé par un homme qui me parlait une langue dont je n'entendais pas un mot ; je conçus cependant, à son empressement, qu'il me donnait quelque avis salutaire [...]. Nous apprîmes que l'île de Gozo était en quarantaine, parce qu'il s'était sauvé un esclave levantin qui s'était caché dans cette île, et que, bien que nous n'y eussions pas abordé, si nous étions aperçus par quelques barques de police, nous serions mis en quarantaine à notre arrivée à Malte. Nous en frémîmes tous ; je craignais plus la quarantaine encore que la $\mathrm{mer}^{55}$.

Ils se dirigèrent vers Malte.

Nous passâmes devant le port de Marsa-Muscet, où nous trouvâmes le vaisseau de guerre français le Caton, qui venait de porter M. de Saint-Priest à Constantinople, et faisait quarantaine entière, attendu que la plus violente peste désolait et dépeuplait toutes les côtes de levant ${ }^{56}$.

Le sentiment de crainte de la quarantaine est partagé par le voyageur anonyme de la Nouvelle relation du voyage et description exacte de l'isle de Malthe qui, en route pour le Levant, fait étape à Malte 105 ans avant Denon. Cet auteur se félicite d'avoir décidé de visiter l'île en route pour, plutôt qu'à son retour du Levant, ce qui lui avait permis d'éviter la quarantaine :

54 Saint-Priest, Mémoires, 10-11.

55 Denon, 136-137.

56 Voir Saint-Priest, Mémoires, $168:$ «[...] on me destina le Caton, vaisseau de ligne de 74 canons » pour le retour à Constantinople en 1778. 
Je suis heureux d'avoir trouvé l'occasion de les [« des choses aussi singulières que celles que j'y (à Malte) ai trouvés et dont je n'étais point informé avant mon arrivée en cette île »] voir en venant, parce qu'au retour de Levant j'aurais trouvé peu de commodités qui y eussent voulu aborder, joint à cela que tous les bâtiments qui en viennent sont obligés de faire quarantaine dans le port de Lazaret, et qu'on ne donne point de pratique aux passagers qu'à même temps on ne la donne à tout l'équipage du navire qui se remet ordinairement à sa route, au premier beau temps, après avoir fait son eau et pris quelque rafraîchissement pour achever son voyage ${ }^{57}$.

Malgré quelques périodes où éclate une épidémie à Malte, faute surtout de négligence ou de manque de sévérité dans l'application des règlements concernant la quarantaine, il faut dire que ces épidémies sont plutôt exceptionnelles. En effet, les voyageurs sont favorablement impressionnés par les mesures sanitaires prises par les autorités maltaises. Sous la date du 4 juin 1770, le voyageur anglais Patrick Brydone décrit la manière dont les autorités portuaires contrôlaient l'entrée des bateaux dans le port de Malte.

L'entrée du port est fort étroite et commandée de deux côtés, par une très grosse forteresse. On nous a hélé de ces deux châteaux; et nous avons été obligés de donner des instructions très détaillées sur notre voyage. En arrivant au côté du quai, un officier du bureau de santé nous a visités, et il nous a contraints de dire par serment d'où nous venons et quel est l'objet de notre expédition. Il s'est comporté de la manière la plus polie, et il nous a envoyé sur le champ M. Rutter, consul de notre nation, pour qui nous avions des lettres de recommandation ${ }^{58}$.

Jean-Marie Roland de la Platière arriva à Malte le 26 novembre 1776 . Il venait de passer une très mauvaise nuit à cause d'une tempête qui fit que lui et ses compagnons abandonnèrent leur bateau à Gozo et firent la traversée pour Malte le lendemain matin. « Un tonnerre affreux qui a roulé toute la nuit et des nuées qui crevaient continuellement sur nos têtes [...]. Vingt fois j'ai cru qu'elle [la barque] serait renversée par la force du vent. " Dans ces conditions on ne s'étonne pas de son impatience pour gagner son hôtel : «A midi nous sommes entrés au port [...]. Après avoir été reconnus par la Santé, ce qui a été long, nous avons été licenciés, et me voici dans une auberge française, servi à la française ${ }^{59}$ ! »

Son tour de Malte comprend une visite au lazaret dont il dit :

La quarantaine se fait à Malte très commodément, dans de grands espaces couverts et découverts ; on cause, on communique, on traite sans le moindre risque. J'ai vu parfumer les lettres et papiers dans une caisse, avec une composition de drogues aromatiques, qu'on y brûle. On donne aux lettres et aux papiers fermés, des coups de ciseaux, afin que la fumée pénètre partout.

Deux ans plus tard arrivèrent deux voyageurs hollandais, accompagnés du peintre

57 Anonyme, Nouvelles relations, 56-57.

58 Brydone, 334-335.

59 Jean-Marie Roland de la Platière, Lettres écrites de Suisse, d'Italie, de Sicile et de Malte (1776-1778) (Amsterdam, 1780), vol. III, 17. 
suisse Louis Ducros, qui font la même expérience :

A 8 heures du matin nous entrâmes heureusement dans le port de Malte [...]. Nous attendîmes une heure avant qu'on vînt nous donner pratique; et celui qui nous la donna alors se trouva être par hasard celui auquel nous étions recommandés, le sieur Poussiergue [Poussielgue], capitaine du port et banquier $[\ldots]^{60}$.

Ils passèrent onze jours à Malte - du 7 au 17 juin 1778 - et font cette observation sur la quarantaine :

Il y a à la Valette deux lazarets, l'un dans le Grand Port sous les remparts de la ville pour ceux qui ne peuvent ou ne veulent pas faire leur quarantaine à bord des vaisseaux qui les ont transportés ; et l'autre dans le Marsa Muscetto au côté occidental de la ville pour les marchandises importées à Malte pour y être vendues. Ces lazarets sont l'un et l'autre très bien entretenus. Comme la quarantaine n'est ordinairement que de vingt jours à Malte, tandis qu'elle est de 40 dans les autres ports de la Méditerranée, la plupart des vaisseaux qui viennent du Levant touchent à cette île pour y faire la quarantaine préférablement à tout autre port, ce qui ne laisse pas que d'être avantageux ${ }^{61}$.

Mais la plus belle description du Lazaret et de ses fonctions, la plus complète, est celle accompagnée d'une planche par Jean-Pierre-Laurent Houel. Ce voyageur fit un voyage en Sicile et à Malte en 1770 et décida de faire un deuxième voyage six ans plus tard. A la suite de ce second séjour de quatre ans, il publia son Voyage pittoresque des isles de Sicile, de Lipari et de Malte. " J'étais peintre et architecte, dit-il dans la Préface, [...] mon voyage pouvait être tout à la fois un récit et une description [...]. J'affirme mes dessins par mes écrits et je confirme mes écrits par mes dessins ${ }^{62} »$.

La planche CCLIV est intitulée Plan de la Barrière et du bureau de la Santé situés au bord de la mer. Elle représente « des chaloupes que l'on suppose aller chercher des marchandises à bord de quelques vaisseaux qui sont à l'ancre au milieu du port et elles viennent les déposer à terre ». S'y joint le dessin de la barrière où se fait le commerce à une distance raisonnable, ce qui assure qu'il n'y a aucun risque de contagion. Le dessin est marqué en divers endroits par des lettres auxquelles fait allusion le texte, intitulé, à son tour, Description et représentation d'une partie du port de Malte qu'on appelle la Barrière et le bureau de la Santé relativement à la quarantaine, et qui décrit la manière dont se passe le marché.

60 Willem Hendrik van Nieuwerkerke and Johan Meerman, éd. Adrian Strickland, Malta from the Hague in the $18^{\text {th }}$ Century, Two Dutch Travel Journals 1778, 1792 (The Hague, 2005), 35.

61 Ibid., 43-44. Cette affirmation est contredite par William Hardman, A History of Malta during the period of the French and British occupations 1798-1815 (London, 1909), 495497, qui constate que le Britannique, W. Eton visita Malte pour la première fois pendant le règne du Grand Maître Rohan (1775-1797) et qu'il passa cinquante jours en quarantaine au lazaret. Voir Cassar, Medical History, 290.

62 Jean Houel, Voyage pittoresque des isles de Sicile, de Lipari et de Malte (Paris, 1778), t. IV, Préface. 
Quelquefois on se contente d'exiger des vaisseaux un simple retard, en les tenant à l'ancre dans le port même de Malte. On permet de débarquer sur la grève les marchandises qui ne s'imprègnent point de la contagion. On accorde aux hommes que l'on n'en croit point attaqués, de mettre pied à terre, et d'entrer dans des avenues marquées par des bornes alignées, qui conduisent au bureau de la santé. Ces bornes sont jointes l'une à l'autre par des monceaux de bois qui en font des allées ; chacune de ces allées a sa destination, soit pour les étrangers, soit pour les habitants du pays. AA : c'est l'endroit du port où l'on dépose les marchandises: ensuite on les porte dans les intervalles $\mathrm{BB}$ et $\mathrm{CC}$. Dans ces intervalles, on y range séparément ces diverses marchandises. Les marchands étrangers se placent dans la barrière DD, d'où ils peuvent traiter avec les marchands du pays, qui sont arrêtés par les barrières EE. Ces barrières qui ont environ cent cinquante pieds de long, peuvent tenir deux mille personnes. Les marchands du pays sont ainsi éloignés des autres par un intervalle de neuf pieds, entre l'avenue $\mathrm{D}$ et celle marquée $\mathrm{E}$ : espace qu'on a reconnu suffisant pour que la contagion ne se communiquât point quand elle n'est pas bien forte, et cet espace n'est pas assez grand pour empêcher qu'on ne puisse voir les marchandises dont on traite.

Quand le temps est doux et serein, les marchés se font ainsi en plein air : mais s'il pleut, ou qu'il fasse trop chaud, ces conférences se font dans un bureau voisin, et dans les cabinets I et L. Les étrangers passent par les magasins $\mathrm{H}$ Je n'en ai pas donné la distribution, parce qu'elle est inutile à mon objet. Dans ces cabinets I et L., les marchands étrangers se placent en II, et confèrent par-dessus l'espace GG qui les sépare d'avec les Maltais placés en L. Leurs affaires finies, ils se retirent par les magasins $\mathrm{H}$, et la porte $\mathrm{M}$.

Le reste de l'espace, au bas de cette estampe, est la continuité du port : la partie qui n'est pas représentée dans cette estampe, sont des magasins, et les demeures de quelques négociants de différentes nations.

Le procès de la fumigation des lettres ne fait pas partie de l'estampe, cependant il est facile de le visualiser grâce à la clarté de la description qu'en fait Houel.

Les papiers, comme les lettres et autres choses qui ne sont pas d'un gros volume et qui ont besoin d'être expédiées promptement, peuvent l'être sans courir de risques. On les passe au feu pour les purifier, et voici comment : d'abord on les fait avec des pinces, et on les perce de deux coups de ciseau, qui les traversent de part en part. On les met ensuite dans une petite armoire, d'environ dix-huit pouces en carré et d'à peu près quatre pieds de haut. Là, sont des grilles de bois qui tiennent lieu de tablettes. Sur ces grilles on met les lettres, les papiers et autres objets qu'on vient purifier.

On brûle au-dessous de la paille dans une grande jatte. Quand elle est en charbon, l'on jette dessus des aromates, composées d'un grand nombre de simples bien mêlés ensemble et parfaitement secs. Ces herbes s'enflamment et brûlent dans l'armoire qu'on ferme aussitôt avec une porte à coulisse. Cette fumigation purifie les papiers dans l'espace d'une demi-heure. Alors ils ne peuvent plus communiquer la peste. Q, est l'espèce de cheminée dans laquelle on brûle la paille.

Mais quand les papiers viennent d'un endroit connu, qui demande une double quarantaine, on commence par tremper les lettres dans le vinaigre, et on leur donne deux 
parfums. L'argent et l'or sont jetés dans un grand bassin plein de vinaigre, cela suffit pour les purifier. Si le vaisseau arrive d'un pays où il $\mathrm{y}$ a la peste, ou voisin d'un pays où elle est, la quarantaine est complète pour les gens de l'équipage, et du double pour le navire et les marchandises.

En conclusion, Houel n'hésite pas à assurer son lecteur que

Toutes les précautions ordonnées par les lois du bureau de la santé sont sévèrement observées, parce que l'on est bien persuadé, que si la peste se répandait dans l'île de Malte, ce serait un peuple perdu. Il n'y viendrait pas de secours : et s'il venait, ils ne seraient peut-être pas reçus. Cette réflexion seule fait trembler ${ }^{63}$.

Concernant le butin humain capturé au cours de la guerre de course soit par les galères de l'Ordre, soit par des corsaires privés, chevaliers ou maltais, l'historien Godfrey Wettinger constate qu'à leur arrivée à Malte, les esclaves étaient soumis à une stricte quarantaine pour la période statutaire dont leurs capteurs n'étaient pas exemptés. Cette période qui durait normalement quarante jours, était souvent raccourcie par le nombre de jours comptés depuis le dernier contact avec la terre, et ce pour vaisseaux ou marchandise, musulmans ou chrétiens, soupçonnés d'infection. C'était le capteur qui encourait les dépenses de la quarantaine. Après quoi, les esclaves étaient vendus aux enchères, sauf que si le capteur était l'Ordre, celui-ci gardait pour les galères les hommes forts et sains. Wettinger donne des exemples de dépenses associées avec la quarantaine. Celles-ci comprenaient la paille pour les esclaves, les droits des certificats issus par les autorités et pour la chambre du Lazaret, la nourriture et l'habillement, le transport des esclaves et les honoraires dus à l'agozzino et à l'interprète ${ }^{64}$. Il arrivait aussi qu'un corsaire maltais déposât ses esclaves au Lazaret et repartît en course. C'était le cas de « la galiotte corsaire maltaise du capitaine Georges Mitrovicz [qui] est arrivée avec sept esclaves [...]. Après les avoir déposés au Lazaret, il est reparti ${ }^{65} »$.

\section{Faits divers}

Il ne serait pas mal à propos de citer quelques faits divers associés à la quarantaine rapportés par trois membres de l'Ordre. Le premier, Gaëtan Reboul était chapelain conventuel maltais dont le journal manuscrit est conservé en partie à la Bibliothèque nationale de Malte. Sous la date du 22 novembre 1748, il parle d'une tempête qui causa le naufrage d'un vaisseau chargé de froment à Gozo. On trouva sept cadavres en mer. Par conséquent, l'île de Gozo fut mise en quarantaine. Cette nouvelle lui était parvenue par une lettre envoyée de Gozo. Celle-ci fut parfumée et coupée « comme si elle venait du Levant ou d'un endroit soupçonné de peste ${ }^{66}$ ». Le même

63 Ibid., 90-92.

64 Voir Godfrey Wettinger, Slavery in the islands of Malta and Gozo ca. 1000-1812 (Malta, 2002), 557-558. L'agozzino surveillait les esclaves. Il devait payer au trésor le prix convenu préalablement pour la perte d'un esclave, due à sa négligence.

65 MAE, CP Malte 16, bulletin du mois d'avril 1779.

66 Vincenzo Laurenza, Giornale de'successi dell'isole di Malta et Gozo - dall'anno 1729 all'anno 1750 - scritto da Gaetano Reboul (Malte, 1939), 106. 
auteur rapporte que le 11 octobre 1744, un matelot anglais fut tué par une sentinelle car il avait désobéi aux ordres et voulut débarquer « avec impertinence ». La peste ravageait Messine et le gouvernement de l'île, prenant toutes les précautions, agissait sévèrement ${ }^{67}$.

Deux autres faits divers se trouvent dans les Mémoires de Pierre Jean-Louis Ovide Doublet, donat ${ }^{68}$ et secrétaire du Grand Maître Ferdinand von Hompesch et, après la cession de l'île à Bonaparte, secrétaire général de la Commission du gouvernement français. Le mémorialiste ne date pas ces deux événements, mais ils devraient se situer en 1783 ou 1784 car il vient de parler du tremblement de terre qui détruisit Messine en 1783. Un bâtiment de commerce français « lequel, venant des échelles du Levant, eut le malheur de voir la peste se manifester en route à son bord et lui enlever en peu de jours plus de la moitié de ses matelots ", se trouvant près de l'île de Lampedusa, y débarqua. L'île était déserte, sauf pour la présence d'un prêtre et six Maltais que l'Ordre entretenait « sous la protection de la France ». Le capitaine Sauzet était le seul à ne pas souffrir de la contagion. Il lui restait deux hommes malades. Il envoya un bateau à Malte avec une lettre au chargé d'affaires de France et une autre au Grand Maître demandant du secours. Le Grand Maître envoya « un chirurgien, quatre gardiens du lazaret et tous les ingrédients nécessaires pour purifier son bâtiment et sa cargaison; et au bout de six semaines il eut la consolation d'entrer sain et sauf, corps et biens dans le port de Malte, purgé de toute contumace, avec ses deux pestiférés radicalement guéris ». Avant de prendre pratique, le capitaine déposa que le même jour où il avait écrit à Malte un corsaire maltais monta à son bord avec des gens de son équipage, s'y enivra et reporta des provisions de d'autres objets, et ce tout en étant averti du danger. Ce corsaire rentra ensuite « dans le port de Marsamuscetto pour y faire sa quarantaine, mais [...] dans sa déposition au lazaret il passa sous silence son débarquement à Lampedusa » et déclara que les 22 hommes de son équipage qu'il avait perdus à cause de la contagion avaient " été tués dans un combat contre un corsaire algérien ». Le corsaire et son lieutenant, ayant affirmé sous serment la vérité de cette déposition furent « condamnés à être pendus comme faussaires, voleurs et parjures et le capitaine eut en sus le poing coupé comme le plus criminel. Tous ceux qui avaient pris part aux vols et aux violences furent punis de la peine des galères perpétuelles ${ }^{69} »$.

Un second récit par le même mémorialiste n'est pas moins étonnant. Comme le premier, il fait preuve de la magnanimité de l'Ordre qui, en conformité avec sa vocation hospitalière, n'hésite pas à subvenir aux besoins des malades, malgré le danger de contagion, et en même temps, à prendre toutes les précautions nécessaires pour éviter une épidémie dans l'île.

67 Ibid., 65.

68 Voir Blondy, L'Ordre de Malte, 14 : «Au dix-huitième siècle, les grands maîtres octroyèrent la demi-croix et le titre de donat à titre de remerciement ou en guise de décoration pour services rendus par des personnes d'humble condition, ou de nationalité maltaise, voire à certains employés de l'Ordre ».

69 Pierre Jean-Louis Ovide Doublet, Mémoires historiques sur l'invasion et l'occupation de Malte par une armée française en 1798 (Paris, 1883), 71-73. 
Un bâtiment vénitien, richement chargé de coton, soieries et drogues du Levant, pour compte de plusieurs négociants sujets du bey de Tunis, entre un matin à pleines voiles, par un vent de nord-est très frais, dans le port de Marsamuscetto, où est situé le lazaret ; et l'on remarque avec surprise qu'il ne paraît pas un seul homme ni sur le pont pour le commandement, ni à la proue pour la manœuvre, et qu'à son passage sous le fort SaintElme, personne ne répond au hélement que le canonnier de garde lui fait avec le portevoix pour savoir d'où il vient, quelle est sa cargaison, s'il a des passagers, des dépêches, etc. : aussitôt le capitaine du port, averti de ces étranges particularités, vogue dans sa barque avec deux gardiens de la santé, pour aller faire à ce bâtiment les questions d'usage. Il le trouve arrêté en face du lazaret, sans avoir jeté l'ancre et portant toutes ses voiles ouvertes, sans que personne paraisse sur le bord. C'est en vain qu'il appelle le capitaine, aucune voix ne répond. Alors il ordonne à l'un des gardiens de monter sur le bâtiment, et d'y observer avec précaution la cause d'un pareil silence. Deux minutes après ce gardien revient dire : "qu'il n'a vu qu'un seul homme dans la couchette du capitaine et presque moribond, qui d'une voix presque mourante lui a dit être resté seul de tout l'équipage ; que le capitaine et tous les autres étant successivement morts de la peste, ils avaient été jetés à la mer, qu'il était resté au timon, tant qu'il en avait eu la force ; que dès qu'il avait aperçu Malte et pu découvrir l'entrée du port, il y avait dirigé la proue ; mais que tombant de lassitude et de besoin, il était descendu dans la chambre du capitaine pour s'y restaurer, chose qu'il n'avait pu faire, le sommeil l'ayant surpris tout à coup et forcé de se coucher; qu'il ignorait depuis quand il était là, mais qu'il se sentait une soif dévorante et voudrait être mort ». Je lui ai donné à boire un verre d'eau, ajouta le gardien, mais sans le toucher en aucune manière.

Là-dessus le capitaine du port, après lui avoir ordonné de rester à bord, de garder le malade à vue, de l'assister de son mieux jusqu'à l'arrivée des secours qu'il allait lui envoyer, et lui avoir défendu sous serment de toucher à rien de suspect, retourna au bureau de santé pour rédiger son rapport par écrit et ensuite le porter au grand maître.

$[\ldots]$

Ce prince lui ordonna de retourner sans délai au bureau de la santé, d'y convoquer les commissaires pour aviser aux premières mesures propres à préserver la santé publique de tout danger, et lui recommanda de ne rien épargner pour sauver, s'il était possible, la vie du seul homme échappé, comme par un prodige, à la destruction qui avait anéanti ses camarades.

Un chirurgien, un autre officier de santé et des gardiens de santé firent débarquer le malade qu'ils transportèrent au lazaret où il fut opéré. Tous ceux qui avaient eu contact avec le malade, s'ils restaient en vie, ne pouvaient «sortir du lazaret qu'au bout de quatre-vingt jours, terme de rigueur fixé pour sa contumace ». Le bâtiment empesté fut brûlé avec sa cargaison ${ }^{70}$. Le mémorialiste tait sur le sort du malade et de ceux qui avaient eu contact avec lui, mais on sait que le chirurgien Stefano Borg mourut longtemps après cet événement.

\section{Conclusion}

Les documents officiels, mémoires et journaux rappellent au lecteur d'aujourd'hui les précautions et mesures exceptionnellement sévères prises par l'Ordre pour s'assurer

$70 \quad$ Ibid., 73-76. 
qu'aucune épidémie n'entrât à Malte, et ce, tout en restant fidèle à sa vocation hospitalière. Quant aux voyageurs, alors que souvent la subjectivité joue un rôle important dans la description des lieux, des monuments et de la vie quotidienne à Malte, de sorte qu'un monument ou un vestige mégalithique passe inaperçu par l'un, alors qu'il est jugé d'une importance primordiale par l'autre; en ce qui concerne la quarantaine, les voyageurs demeurent d'accord sur l'accueil réservé aux passagers et aux vaisseaux qui entraient dans le port de Malte. Leurs remarques sur la stricte observance des règlements gouvernant la quarantaine confirment les constations des documents d'archives. Lorsque Bonaparte, en route pour l'Egypte, prit Malte en juin 1798 et chassa l'Ordre, il fit dans les six jours que dura sa présence sur l'ile beaucoup de changements. Quant à la quarantaine, les 29 articles approuvés le 22 thermidor an 6 par la Commission du gouvernement renforcèrent pour l'essentiel les règlements autrefois promulgués par l'Ordre, plusieurs articles commençant par ou comprenant la phrase : «il y aura comme pour le passé », ou « comme ci-devant» ou « comme autrefois » ${ }^{71}$. Dernier vestige, il reste encore en place aujourd'hui quelques-unes des bornes en pierres qui servaient à soutenir les monceaux de bois dont l'ensemble démarquait « la barrière » qui séparait les étrangers des habitants du pays ${ }^{72}$, témoins silencieux d'un passé toujours en éveil contre

Un mal qui répand la terreur, $[\ldots]$

La peste (puisqu'il faut l'appeler par son nom) ${ }^{73}$.

71 NLM, Arch 6523b, 361-367. Par exemple, article 4: « Il y aura comme pour le passé, 24 bateliers pour les 12 barques destinées aux besoins du bâtiment en quarantaine et du Lazaret », ou l'article 9 : «Le médecin visitera les bâtiments qui lui sont désignés et traitera les malades comme pour le passé ».

72 Voir The Times (Malta), 26 August 2008, 11. "One World-Protecting the most significant buildings, monuments and features of Valletta (42) New Fish Market at the Barriera ». The site of the new fish market was the site of the former Barriera (barrier). This "barrier" was installed during the period of the Knights of St. John to quarantine those persons arriving by sea from any acquaintances who visited them while in quarantine. The quarantine area was demarcated by a barrier consisting of wooden barriers fitted in stone pillars and a few of these stone pillars may still be seen in the area. At the Barriera a new fish market was built in 1937. The new fish market was partly destroyed during World War II and the damage reconstructed soon after the end of hostilities and is still used for the same purpose. L'autre établissement de Manoel Island continua sa fonction d'isolement sous la domination britannique (1800-1964) de l'île, accueillant, parmi d'autres, Benjamin Disraeli en 1830, Lord Byron en 1811, Sir Walter Scott en 1831, Rev. (later Cardinal) John Henry Newman en 1833, William Tackeray en 1844, Alphonse de Lamartine en 1832 et le peintre Horace Vernet en 1840 .

Jean de La Fontaine, Fables (Paris, 1678-1679), VII, 1, Les Animaux malades de la Peste. 